\title{
Experimental verification of the usefulness of the $n$th power law MOSFET model under hot carrier wearout
}

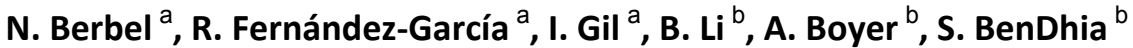 \\ a Electronic Engineering Department, UPC Barcelona Tech., Colom 1, 08222 Terrassa, Spain \\ b Départment du Genie Electrique et Informatique, INSA de Toulouse, Avenue de Rangueil \\ 135, 31077 Toulouse, France
}

\begin{abstract}
In this paper the usefulness of the $n$th power law MOSFET model under Hot Carrier Injection $(\mathrm{HCl})$ wearout has been experimentally demonstrated. In order to do that, three types of nFET transistors have been analyzed under different $\mathrm{HCl}$ conditions and the $n$th power law MOSFET model has been extracted for each sample. The results show that the model can reproduce the MOSFET behavior under $\mathrm{HCl}$ wearout mechanism. Therefore, the impact of $\mathrm{HCl}$ on circuits can be analyzed by using the $n$th power law MOSFET model.
\end{abstract}

\section{Introduction}

The $n$th power law MOSFET model, also namely Sakurai-Newton model, is a wellknown analytical model, which can reproduce the MOSFET behavior with only six parameters [1]. The model is expressed in the following equation:

$$
\begin{aligned}
& V_{\mathrm{DSAT}}=K \cdot\left(V_{\mathrm{GS}}-V_{\mathrm{TH}}\right)^{m} \\
& I_{\mathrm{DSAT}}=B \cdot\left(V_{\mathrm{GS}}-V_{\mathrm{TH}}\right)^{n} \\
& F_{\text {GS }}<F_{\text {TU }} \quad F_{D}=0 A
\end{aligned}
$$

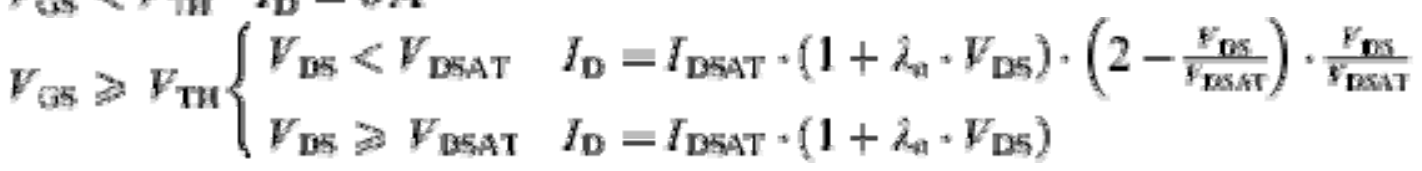

where $V_{G S}$ and $V_{D S}$ are the gate-to-source and the drain-to-source voltages, respectively. $V_{T H}$ denotes the threshold voltage, $V_{\mathrm{DSAT}}$ the drain saturation voltage and $I_{\text {DSAT }}$ the drain saturation current. Parameters $K$ and $m$ control the linear region characteristics whereas $B$ and $n$ determine the saturated region characteristics. $\lambda$ is related to the finite drain conductance in the saturated region. The simplicity of the model allows an analytical processing. The model (or models derived from it) has been extensively used in order to evaluate the behavior of several CMOS circuits [1], [2], [3] and [4] due to its simplicity. It should be pointed out that the $n$th power law MOSFET parameters correspond to an analytical non-physical model and it is only valid for voltage gates above the voltage threshold. 
Hot Carriers Injection $(\mathrm{HCl})$ is the phenomenon in solid-state or semiconductor electronic devices where either an electron or a "hole" gains sufficient kinetic energy to overcome a potential barrier necessary to break an interface state [5]. $\mathrm{HCl}$ does not always lead to circuit failure [6], but provokes undesired effects such as the reduction of the speed at which the transistor can operate. Therefore, to determine how sensitive are the electronic circuits to $\mathrm{HCl}$, some accurate models are required. In this sense, MOSFET models, which take into account the effects of $\mathrm{HCl}$, and that could be implemented in circuit simulators are required. Equivalent electrical circuits for FETs under $\mathrm{HCl}$ have been already presented, which can be included in SPICE simulators. In this approach, additional components are needed [7]. An alternative approach is the description of the $\mathrm{HCl}$ MOSFET using models like, for example, BSIM, whose parameters are extracted after the $\mathrm{HCl}$ stress. This approach would be the most accurate, but a large number of parameters and a complex extraction process are required. In this work, and alternative approach has been followed, and the usefulness of $n$th power law MOSFET model under $\mathrm{HCl}$ effect has been demonstrated experimentally. Moreover, the impact of $\mathrm{HCl}$ wearout on each Sakurai-Newton model parameters has been experimentally obtained.

\section{Experimental}

To analyze the impact of the $\mathrm{HCl}$ in the nMOS, a Keithley 2602A Source-meter, a digital multimeter and an acquisition board have been used, all of them controlled with a laptop (Fig. 1). First of all, it is necessary to obtain the stress conditions. The $V_{D S}$ stress voltage ( $V_{\text {STRESS }}$ ) should be equal to the drain-to-source voltage breakdown minus $0.5 \mathrm{~V}$. The $V_{G S}$ stress voltage is obtained by measuring the substrate current (ISUB $)$ whereas the $V_{G S}$ is swept with the $V_{D S}$ set to $V_{\text {STRESS. The }} V_{G S}$ stress voltage is defined as the gate voltage corresponding to the maximum $I_{S U B}$ value on the $I_{S U B}-V_{G S}$ curve. Table 1 summarizes the different stress voltages applied to the different type of nMOS transistors.

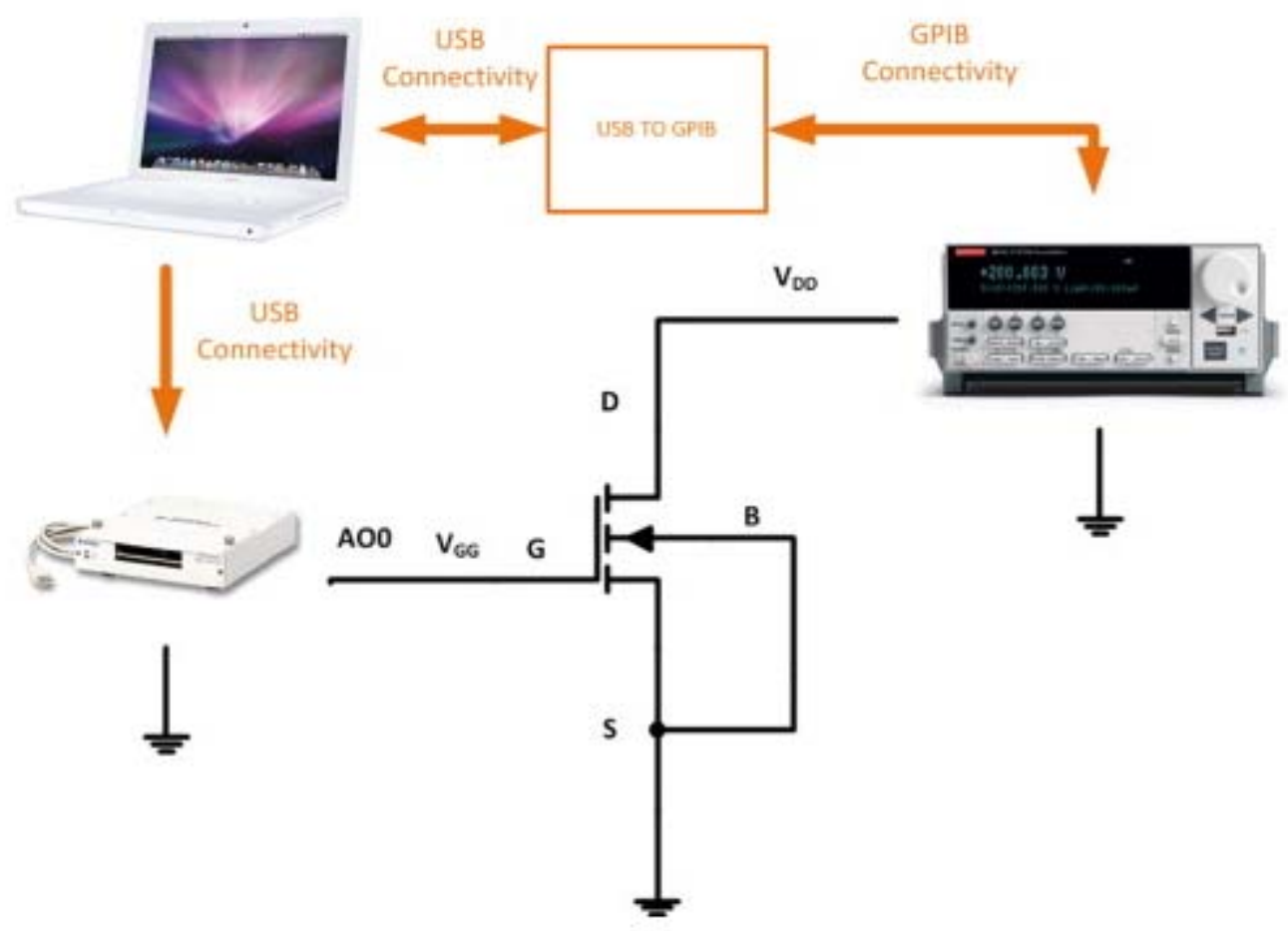


Fig. 1. Set-up to obtain the impact of the $\mathrm{HCl}$ on $\mathrm{nMOS}$.

Table 1. Type of transistor measured and stress voltages applied.

\begin{tabular}{|c|c|c|}
\hline & $\left.V_{\text {DS }}\right|_{\text {STRESS }}(\mathrm{V})$ & $\left.V_{\text {GS }}\right|_{\text {STRESS }}(\mathrm{V})$ \\
\hline \multirow[t]{2}{*}{ High voltage long $(W=10 \mu \mathrm{m} L=1.52 \mu \mathrm{m})$} & 7 & 2.6 \\
\hline & 5 & 1.9 \\
\hline \multirow[t]{2}{*}{ High voltage short $(W=10 \mu \mathrm{m} L=0.38 \mu \mathrm{m})$} & 7 & 2.05 \\
\hline & 5 & 1.6 \\
\hline \multirow[t]{2}{*}{ Low voltage long $(W=10 \mu \mathrm{m} L=0.4 \mu \mathrm{m})$} & 3 & 0.97 \\
\hline & 2.5 & 0.9 \\
\hline
\end{tabular}

\section{Results}

Fig. 2 and Fig. 3 show the ID-VDS and the ID-VGS curve characteristic, respectively, for several stress time. As it is observed, the drain saturation current is reduced due to the effect of the $\mathrm{HCl}$. Fig. 4 and Fig. 5 plot the fitting of the measurements and the Sakurai-Newton MOSFET model for the fresh component and after $700 \mathrm{~s}$ of stress. A good correlation between the mathematical model and the measurements has been obtained. Therefore, the impact of $\mathrm{HCl}$ wearout on each $n$th power law MOSFET model has been evaluated and it is shown below.

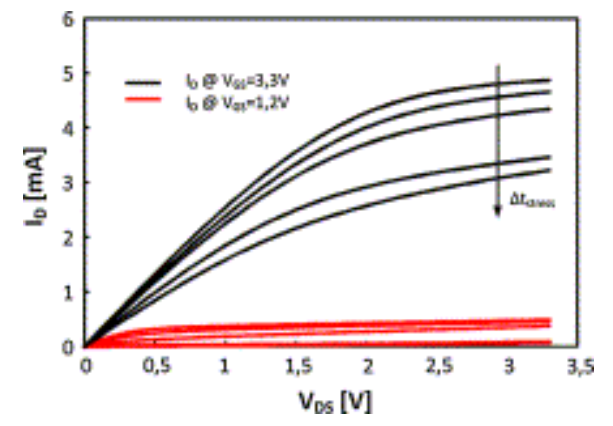

Fig. 2. $I_{D}-V_{D S}$ for several stress time and several $V_{G S}$.

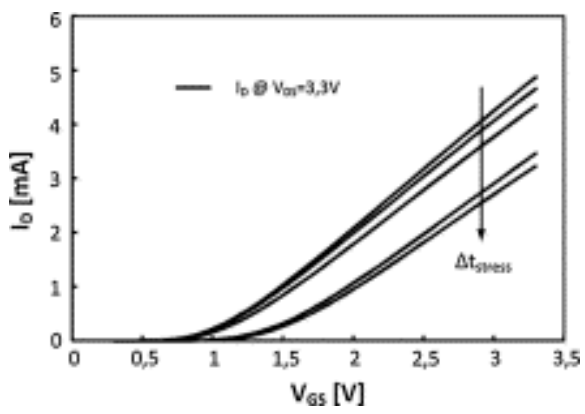

Fig. 3. $I_{D}-V_{G S}$ for several stress time. 


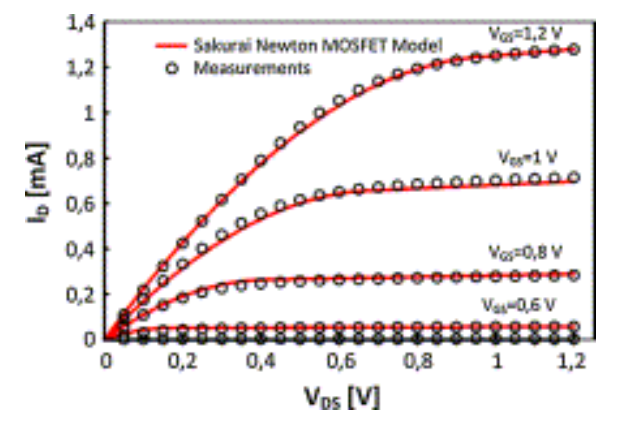

Fig. 4. Comparison between the measurements and the Sakurai-Newton MOSFET model for the fresh component.

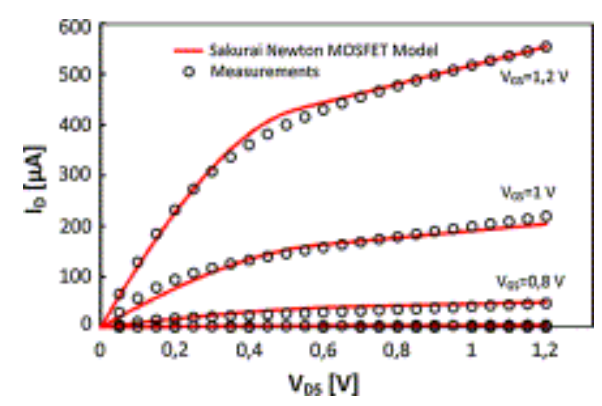

Fig. 5. Comparison between the measurements and the Sakurai-Newton MOSFET model for the $t_{\text {STRESS }}=700 \mathrm{~s}$.

Fig. 6 depicts the increase of the finite drain conductance $(\lambda)$, which produces a change in the slope of the drain current (in the saturated region), as can be observed in Fig. 2. Fig. 7 plots the threshold voltage shift, which is more significant on the high voltage short transistor, rather than on the high voltage long and low voltage long. The VTH enhancement provokes a drain current decrease, as can be observed on Fig. 3 . The $n$ parameters shift is plotted in Fig. 8, which corresponds to a value less than $10 \%$ for the three types of nMOS transistors. On the other hand, B parameter shift is plotted in Fig. 9 , which decreases as long as the stress time increases. This parameter is related with the transconductance of the device. Fig. 10 and Fig. 11 show the $\mathrm{K}$ and $\mathrm{m}$ parameters shift, related with control of the linear region. Both parameters are modified due to the change of the linear ID-VDS curve characteristic, as seen in Fig. 4 and Fig. 5.

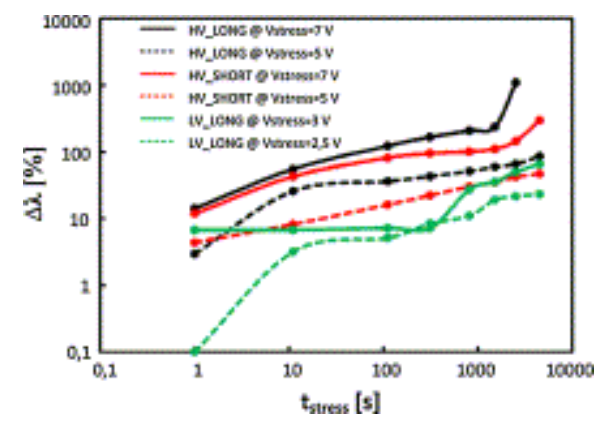

Fig. 6. Variation of the finite drain conductance $(\lambda)$ versus the stress time. 


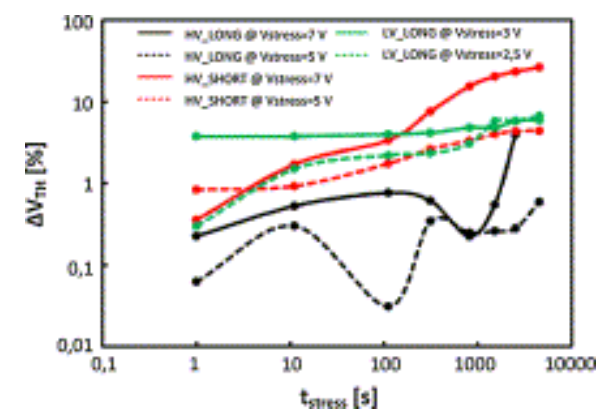

Fig. 7. Variation of the threshold voltage versus the stress time.

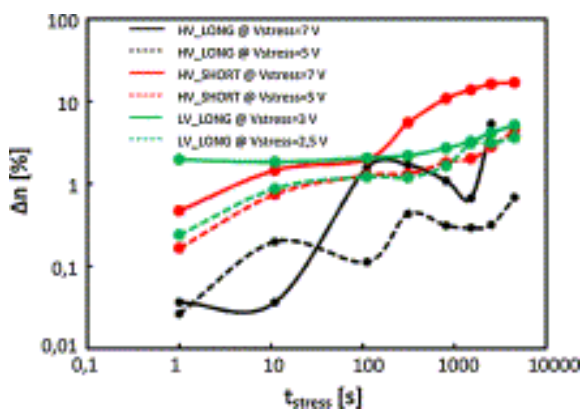

Fig. 8. Variation of the $n$ parameter versus the stress time.

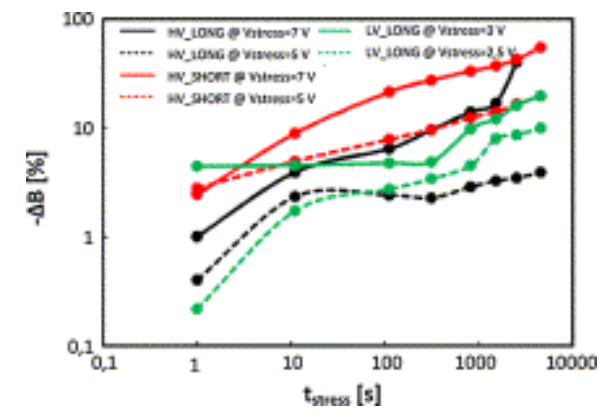

Fig. 9. Variation of the transconductance $(B)$ versus the stress time.

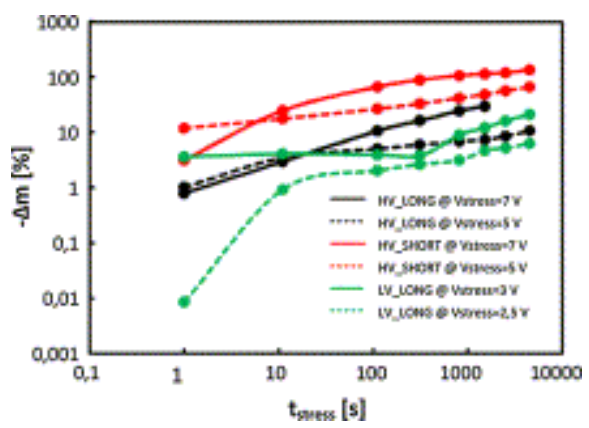

Fig. 10. Variation of the $m$ parameter versus the stress time. 


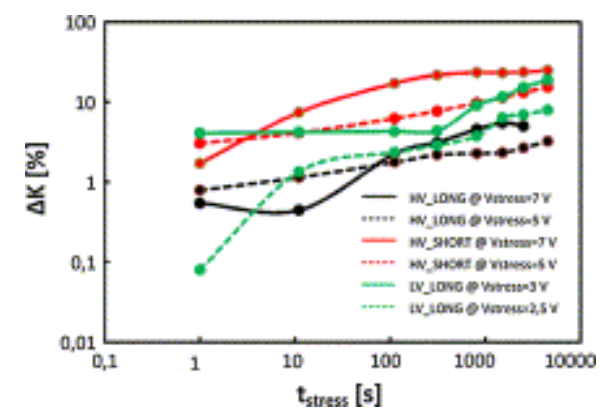

Fig. 11. Variation of the $K$ parameter versus the stress time.

\section{Conclusions}

In this paper, the usefulness of the Sakurai-Newton MOSFET model under $\mathrm{HCl}$ wearout has been experimentally demonstrated for different nFET transistors and different stress conditions. The impact of $\mathrm{HCl}$ on each parameter has been also analyzed. The results show that the models can be useful in order to fit the MOSFET behavior under $\mathrm{HCl}$ stress and it can be included in a circuit simulator to predict the impact of $\mathrm{HCl}$ on digital circuit behavior, where the sub-threshold behaviors are not so important.

\section{Acknowledgements}

This work has been supported by the Spain-MICINN under Projects TEC2010-18550 and AGAUR 2009 SGR 1425. The authors want to thank to B. Vrignon from Freescale Semiconductors for sample provision.

\section{References}

[1] T. Sakurai and A.R. Newton, A simple MOSFET model for circuit analysis. IEEE Trans Electr Dev, 38 April (1991), pp. 887-894

[2] J.L. Rossello and J. Segura, An analytical charge-based compact delay model for submicrometer CMOS inverters. IEEE Trans Circuits Syst I: Regular Papers, 517 (2004), pp. 1301-1311

[3] R. Fernandez, R. Rodriguez, M. Nafria and X. Aymerich, DC broken down MOSFET model for circuit reliability simulation. Electr Lett, 416 (2005), pp. 368-370

[4] Chandra Nishant, Yati Apoorva Kumar, Bhattacharyya AB. Extended-Sakurai-Newton MOSFET Model for Ultra-Deep-Submicrometer CMOS Digital Design, vlsid. In: 2009 22nd international conference on VLSI design; 2009. p. 247-52..

[5] A. Acovic, G. La Rosa and Y. Sun, A review of hot-carrier degradation mechanisms in MOSFETs. Microelectr Reliab, 368 (1996), pp. 845-869.

[6] Xiaojun Li, Jin Qin, Bing Huang, Xiaohu Zhang and J.B. Bernstein, SRAM circuit-failure modeling and reliability simulation with SPICE. IEEE Trans Device Mater Reliab, 62 (2006), pp. 235-246.

[7] Xiaojun Li, Jin Qin and J.B. Bernstein, Compact modeling of MOSFET wearout mechanisms for circuit-reliability simulation. IEEE Trans Device Mater Reliab, 81 (2008), pp. 98-121 